\title{
RECENT STAR FORMATION IN THE EXTREME OUTER DISK OF M83
}

\author{
David A. Thilker, ${ }^{1}$ Luciana Bianchi ${ }^{1}$ Samuel Boissier, ${ }^{2}$ Armando Gil de Paz, ${ }^{2}$ Barry F. Madore, ${ }^{2}$ \\ D. Christopher Martin, ${ }^{3}$ Gerhardt R. Meurer, ${ }^{1}$ Susan G. Neff, ${ }^{4}$ R. Michael Rich, ${ }^{5}$ David Schiminovich, ${ }^{3}$ \\ Mark Seibert, ${ }^{3}$ Ted K. Wyder, ${ }^{3}$ Tom A. Barlow, ${ }^{3}$ Yong-Ik Byun, ${ }^{6}$ Jose Donas, ${ }^{7}$ Karl Forster, ${ }^{3}$ Peter G. Friedman, ${ }^{3}$ \\ Timothy M. Heckman, ${ }^{8}$ Patrick N. Jelinsky, ${ }^{9}$ Young-Wook Lee, ${ }^{6}$ Roger F. Malina, ${ }^{7}$ Bruno Milliard, ${ }^{7}$ \\ Patrick Morrissey, ${ }^{3}$ Oswald H. W. Siegmund, ${ }^{9}$ Todd Small, ${ }^{3}$ Alex S. Szalay, ${ }^{8}$ and Barry Y. Welsh ${ }^{9}$ \\ Received 2004 May 4; accepted 2004 August 23; published 2005 January 17
}

\section{ABSTRACT}

Ultraviolet imaging with the Galaxy Evolution Explorer (GALEX) has revealed an extensive sample of UVbright stellar complexes in the extreme outer disk of M83, extending to about 4 times the radius at which the majority of $\mathrm{H}$ II regions are detected $\left(R_{\mathrm{H} \text { I }}=5 ! 1\right.$, or $\left.6.6 \mathrm{kpc}\right)$. These sources are typically associated with largescale filamentary H I structures in the warped outer disk of M83 and are distributed beyond the galactocentric radii at which molecular interstellar medium has yet been detected. We present measured properties of these stellar complexes, including far-UV and near-UV magnitudes and local gas surface density. Only a subset of the outer-disk UV sources have corresponding $\mathrm{H}$ II regions detected in $\mathrm{H} \alpha$ imaging, consistent with a sample of mixed age in which some sources are a few megayears old and others are much more evolved $\left(\sim 10^{8} \mathrm{yr}\right)$.

Subject headings: galaxies: formation — galaxies: individual (M83) — galaxies: ISM

Online material: color figures

\section{INTRODUCTION}

The Galaxy Evolution Explorer (GALEX), with its 1.25 field of view and sensitivity to stellar populations younger than a few hundred megayears, is well suited for addressing the topic of star formation at large galactocentric radii. Recent star formation within such environments has been detected in $\mathrm{H} \alpha$ and broadband observations of a few galaxies (NGC 628, NGC 1058, NGC 6946: Ferguson et al. 1998b, also Lelievre \& Roy 2000 for NGC 628; M31: Cuillandre et al. 2001; NGC 6822: de Blok $\&$ Walter 2003). One goal of the GALEX Nearby Galaxy Survey (NGS; Bianchi et al. 2005a) is to conduct a census of outer-disk star formation in a statistically significant sample of galaxies.

The confirmed occurrence of star formation in the outer disk of ordinary spiral galaxies has far-reaching implications. First, if massive stars are forming at large radii, then the outer disk continues to undergo chemical enrichment. This may clarify the origin of dust detected in the extended $\mathrm{H}$ I disks of spiral galaxies (Popescu \& Tuffs 2003). Secondly, radiative and mechanical feedback processes associated with massive stars are expected to be more efficient in the comparatively rarified interstellar medium (ISM) and uncrowded environment at large radii. In particular, the unresolved issue of $\mathrm{H}$ I production via $\mathrm{H}_{2}$ dissociation (Smith et al. 2000; Allen et al. 1986) can be studied in detail.

\footnotetext{
${ }^{1}$ Center for Astrophysical Sciences, Johns Hopkins University, 3400 North Charles Street, Baltimore, MD 21218; dthilker@pha.jhu.edu.

${ }^{2}$ Observatories of the Carnegie Institution of Washington, 813 Santa Barbara Street, Pasadena, CA 91101.

${ }^{3}$ California Institute of Technology, MC 405-47, 1200 East California Boulevard, Pasadena, CA 91125.

${ }^{4}$ Laboratory for Astronomy and Solar Physics, NASA Goddard Space Flight Center, Greenbelt, MD 20771.

${ }^{5}$ Department of Physics and Astronomy, University of California, Los Angeles, CA 90095.

${ }^{6}$ Center for Space Astrophysics, Yonsei University, Seoul 120-749, Korea.

${ }^{7}$ Laboratoire d'Astrophysique de Marseille, BP 8, Traverse du Siphon, 13376 Marseille Cedex 12, France.

${ }^{8}$ Department of Physics and Astronomy, Johns Hopkins University, Homewood Campus, Baltimore, MD 21218.

${ }^{9}$ Space Sciences Laboratory, University of California at Berkeley, 601 Campbell Hall, Berkeley, CA 94720.
}

The presence of recently formed stellar complexes at large galactocentric radii also provides a simplified laboratory for investigating the star formation threshold - the (minimum) gas column density required for star formation to occur spontaneously. The precise workings of this mechanism remain a matter of debate. Kennicutt (1989) demonstrated that star formation is rare in disk environments having total gas surface density $\Sigma_{\text {gas }}<$ 5-10 $M_{\odot} \mathrm{pc}^{-2}$. Martin \& Kennicutt (2001) showed the observed threshold gas density varies by about an order of magnitude at the "edge" of the star-forming disk $\left(R=R_{\mathrm{H}}\right)$ in different galaxies, but the ratio of $\Sigma_{\text {gas }}$ to a critical density (which depends on the physical and dynamical conditions of each galaxy in particular) is approximately constant at this position.

Deep H $\alpha$ imaging by Ferguson et al. (1998b) and Lelievre \& Roy (2000) showed that star formation regions only a few megayears old exist beyond two optical radii $\left(R_{25}\right)$ in NGG 628, NGC 1058, and NGC 6946. Lelievre \& Roy (2000) demonstrated that the $\mathrm{H}$ II region luminosity function (LF) slope is significantly steeper in the outer disk relative to a galaxy-wide sample, possibly indicating a genuine change in the initial cluster mass function $(\mathrm{CMF})$, or a population that is preferentially older. Ferguson et al. (1998b) noted that the outer H II regions appear small, isolated, and faint compared to inner-disk analogs.

In this Letter, we present far-UV (FUV) and near-UV (NUV) wide-field GALEX imagery that reveals an extensive population of recent star-forming regions in the extreme outer disk of M83, out to $\sim 3$ times the Holmberg radius (7'3). Some of these sources were discovered by Wamsteker et al. (1983) in deep red (12704+RG 630) plates, but observed properties were never published. We analyze the GALEX data in conjuction with visible and $21 \mathrm{~cm}$ imagery to place the outer-disk sources in context relative to the underlying stellar population and extended $\mathrm{H}$ I disk. The GALEX data are of particular importance, because it demonstrates that $\mathrm{H} \alpha$ observations will fail to detect a significant population of moderate-age clusters in the outermost disk of spiral galaxies, leading to an underestimate of galactic evolution in these locales. Processes such as chemical enrichment (Ferguson et al. 1998a), disk heating (Sellwood \& Balbus 1999), and ISM phase balance 


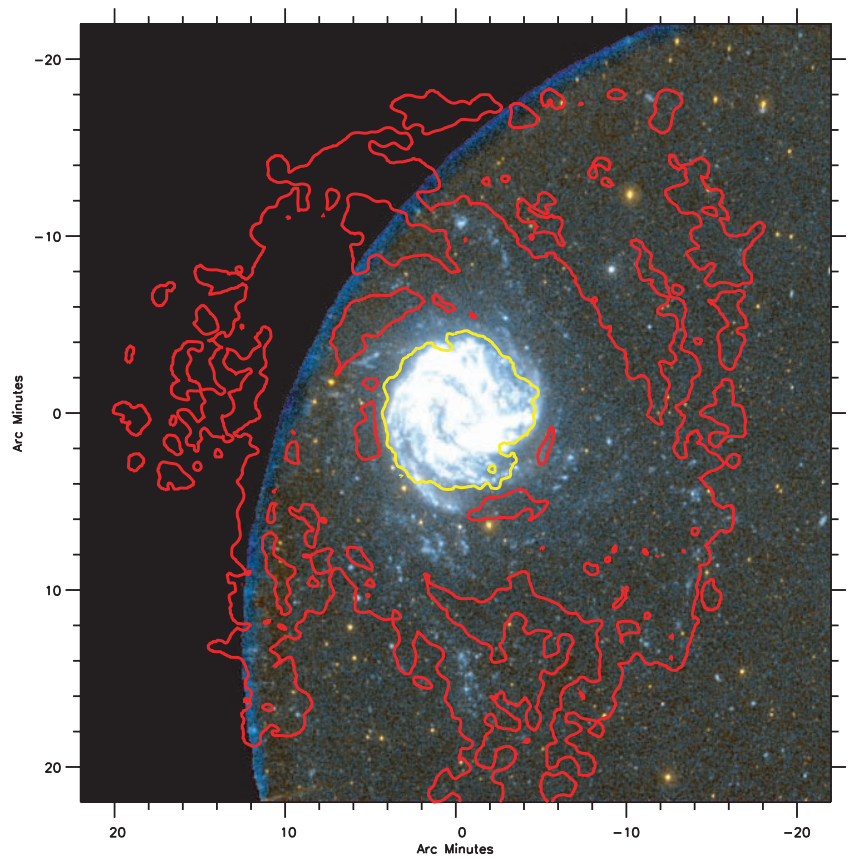

FIG. 1.-GALEX FUV and NUV color-composite image of M83, highlighting the newly rediscovered sites of recent star formation in the extreme outer disk. We show FUV in blue and NUV in red (along with their average in green). A red contour represents the extent of the $\mathrm{H}$ I distribution detected by Tilanus \& Allen (1993). The blue $\mathrm{H}$ I contour is drawn at $1.8 \times 10^{20} \mathrm{~cm}^{-2}$, corresponding to $\sim 0.4 M_{\odot} \mathrm{pc}^{-2}$. We also include a yellow contour from Crosthwaite et al. (2002), indicating total neutral gas surface density, $\Sigma_{\text {gas }}$ of $10 M_{\odot} \mathrm{pc}^{-2}$. The yellow $\Sigma_{\text {gas }}$ contour lies immediately within $R_{\mathrm{H} \text { I }}$. The projected spatial extent of the field $\left(44^{\prime}\right)$ is $57.6 \mathrm{kpc}\left(R_{\mathrm{HI}}=6.6 \mathrm{kpc}\right)$ at a distance of $4.5 \mathrm{Mpc}$.

(Allen et al. 1986) may be significantly influenced, given that the (predominantly) B star population traced by GALEX effectively drives these feedback mechanisms. GALEX NGS observations show that M83 is not unique in terms of outer-disk UV morphology. We will analyze UV-visible-IR outer-disk properties of a representative galaxy sample in a subsequent paper.

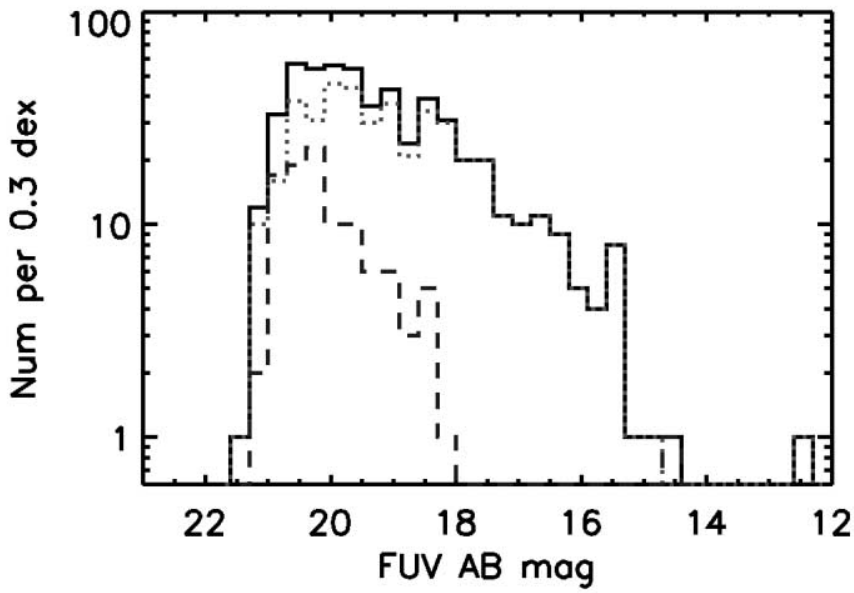

FIG. 2.-GALEX FUV LFs of the UV-bright stellar complexes in M83 having photometric errors $<0.25 \mathrm{mag}$. Image shows the FUV LF for all sources (solid line), inner-disk $\left(R<R_{\mathrm{H}}\right)$ sources (dotted line), and outer-disk $\left(R \geq R_{\mathrm{H}}\right)$ sources (dashed line). Outer-disk complexes appear typically fainter and have a steeper slope in the FUV LF, relative to inner-disk objects, although detailed assessment of completeness and blending is needed to bolster these statements. [See the electronic edition of the Journal for a color version of this figure.]

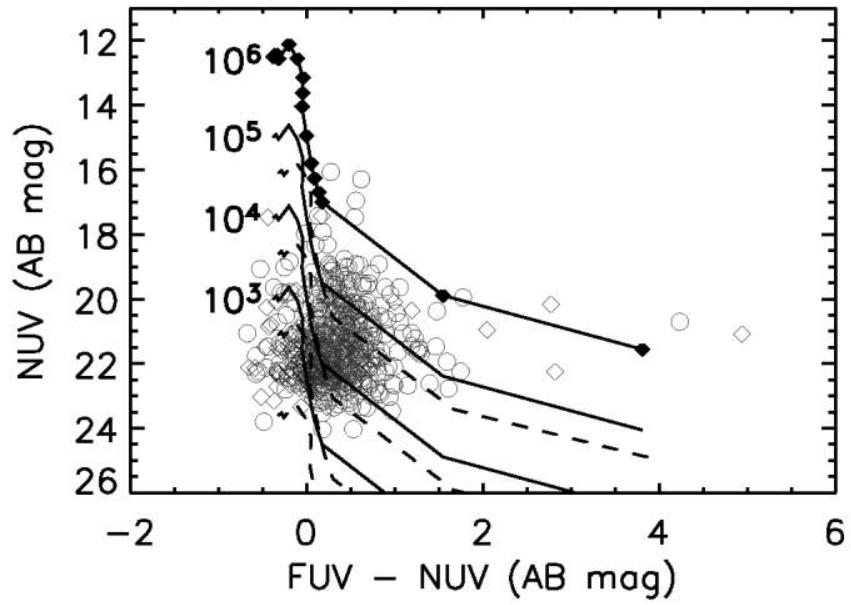

FIG. 3.-GALEX (FUV - NUV, NUV) CMD for M83's UV-bright stellar complexes (same sample and radial classification as Fig. 2). Inner-disk sources $\left(R<R_{\mathrm{H} \mathrm{I}}\right)$ are plotted with circles, whereas outer-disk sources $\left(R \geq R_{\mathrm{H}}\right)$ are indicated with diamonds. Our data points have been corrected only for Galactic foreground extinction, $E(B-V)=0.066$. The black curves show model predictions for starburst populations of $10^{3}, 10^{4}, 10^{5}$, and $10^{6} M_{\odot}$ as a function of age $(\log$ age $=5.7,6.0,6.3,6.5,6.6,6.75,7.0,7.3,7.6,7.75,7.9,8.0,8.7,9.0$ indicated for $\left.10^{6} M_{\odot}\right)$. Dashed lines indicate the same models reddened with $E(B-V)=0.5$, a value slightly higher than the median $E(B-V)$ determined for a large sample of clusters in M83 (Harris et al. 2001). [See the electronic edition of the Journal for a color version of this figure.]

\section{OBSERVATIONS AND DATA ANALYSIS}

M83 (NGC 5236) was observed with GALEX on 2003 June 7 for a single orbital night as part of the NGS, with an exposure of

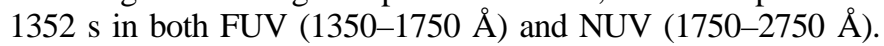
Figure 1 shows the color-composite GALEX image of M83. Two bright stars eastward of M83 prevented us from centering the target in the field, but our data includes most of the outer disk. The rms

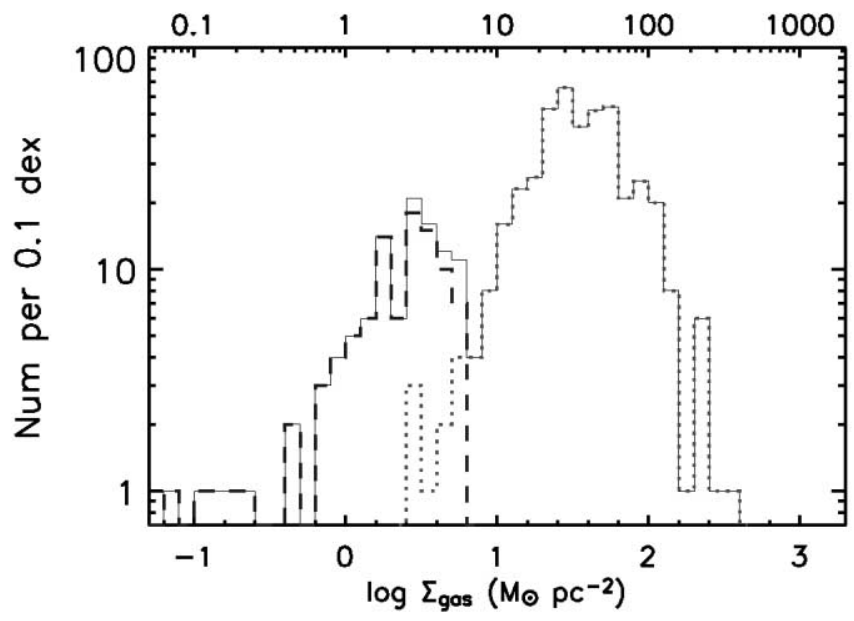

FIG. 4.-Local $\Sigma_{\text {gas }}$ at sites of recent star formation in M83, evaluated at $1.1 \mathrm{kpc}\left(50^{\prime \prime}\right)$ resolution. The overall distribution (solid histogram) appears largely bimodal, with inner-disk UV sources (within $R_{\mathrm{H} \text { II }}$, dotted histogram) forming in locales with total surface density greater than $\sim 10 M_{\odot} \mathrm{pc}^{-2}$. Recently formed outerdisk stellar complexes (dashed histogram) exist within environments characterized by a local surface density about an order of magnitude smaller, although the $\mathrm{CO}$ map used to compute the $\mathrm{H}_{2}$ contribution does not extend to large galactocentric radii. High-resolution $\mathrm{H} \mathrm{I}$ and $\mathrm{CO}$ observations toward the outer UV-bright complexes are critically needed. Such data would enable us to investigate $\mathrm{H}$ I production via $\mathrm{H}_{2}$ photodissociation (Smith et al. 2000) as well as to make a fundamental check on the star formation threshold (Kennicutt 1989). We reiterate that this plot shows local measurements of $\Sigma_{\text {gas }}$, whereas Fig. 5 presents an azimuthally averaged surface density as a function of galactocentric radius. 


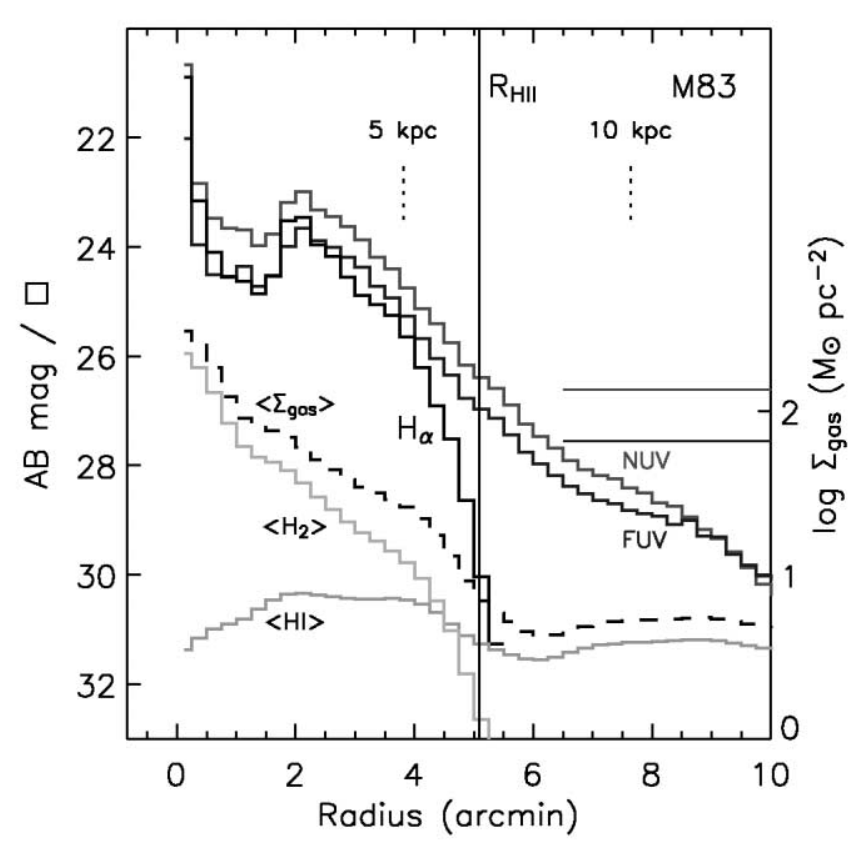

FIG. 5.- Radial profiles of the median FUV, NUV, and $\mathrm{H} \alpha$ surface brightness in M83, plus average surface densities derived from $\mathrm{H}$ I and CO data. Azimuthally averaged $\Sigma_{\text {gas }}$ is shown with a dashed line. The UV profiles are presented in absolute units, while the $\mathrm{H} \alpha$ profile is arbitrarily normalized. We indicate $R_{\mathrm{H} \text { I }}$ with a vertical line, and the UV sky background with horizontal lines. Slight deviations from flatness across the GALEX field, coupled with the fact that M83 is positioned on the edge of the field, make it impractical to follow the profiles beyond $\sim 10^{\prime}$. Note the remarkable difference between the $\mathrm{H} \alpha$ and UV profiles at (subcritical) galactocentric radii $>5 \mathrm{kpc}$. [See the electronic edition of the Journal for a color version of this figure.]

sensitivity is 26.6 (26.8) AB mag for FUV (NUV), or 27.5 (27.6) $\mathrm{AB}$ mag $\operatorname{arcsec}^{-2}$, expressed in units of surface brightness evaluated at the scale of the point-spread function (PSF).

Boissier et al. (2005) also analyze the GALEX M83 data set, emphasizing the inner disk in regard to extinction and chemical evolution models. Courtesy of P. Crosthwaite, we gained access to the $21 \mathrm{~cm}$ VLA observations discussed by Tilanus \& Allen (1993). For the inner $14^{\prime} \times 14^{\prime}$ of M83, we also used the CO map of Crosthwaite et al. (2002) to estimate $\Sigma_{\text {gas }}$ as a function of position. $\mathrm{H} \alpha$ and $R$-band imaging was obtained using the Swope $1 \mathrm{~m}$ telescope at Las Campanas Observatory.

We adopt a distance of $4.5 \mathrm{Mpc}$ to M83 (Thim et al. 2003); thus, the $\sim 5^{\prime \prime}$ GALEX PSF corresponds to $110 \mathrm{pc}$. Accordingly, our detections in M83 are generally not single clusters, but rather aggregates possibly consisting of a few (unresolved) clusters.

Photometry of the discrete UV sources within M83 was accomplished using Source Extractor (SExtractor; Bertin \& Arnouts 1996) to define an isophotal aperture for every object detected within our NUV image. These irregular-region boundaries were then applied to both the NUV and FUV data for the measurement of background-subtracted source flux, after applying a distortion correction to the FUV image to ensure registration with our NUV data. The background for every source was estimated locally (within $\sim 250 \mathrm{pc}$ ). We measured median surface brightness as a function of galactocentric radius as described by Bianchi et al. (2005b), with $i=25^{\circ}$ and P.A. $=226^{\circ}$ (Crosthwaite et al. 2002).

\section{RECENT STAR FORMATION IN THE OUTER DISK OF M83}

M83 is considered prototypical of those galaxies showing a well-defined edge of the star-forming disk (e.g., Martin \& Ken-

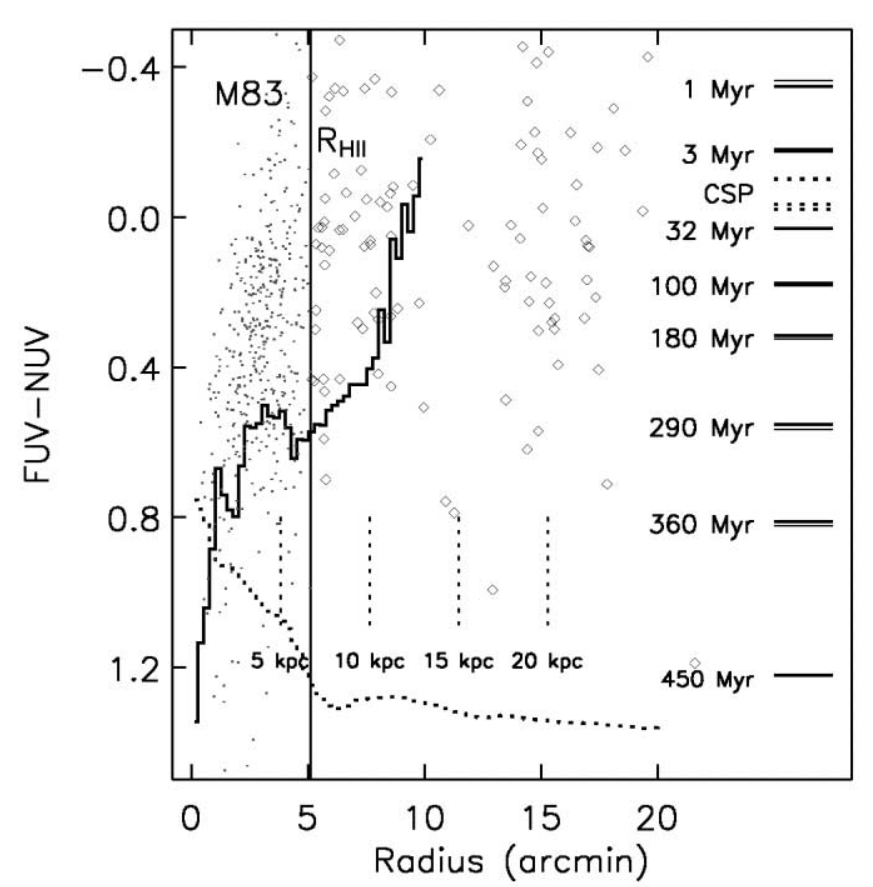

FIG. 6.-Median FUV - NUV as a function of galactocentric radius, with individual inner (dots) and outer (diamonds) stellar complexes plotted for comparison. We also show the FUV - NUV color for model starburst populations of varied age and three different periods of continuous star formation (CSP: $100 \mathrm{Myr}, 1 \mathrm{Gyr}$, and $10 \mathrm{Gyr})$. Intrinsic and reddened $[E(B-V)=$ $0.2]$ colors are indicated with thick and thin line segments, respectively, although the difference is minimal compared to the observed run of FUV NUV. The $\left\langle\Sigma_{\text {gas }}\right\rangle$ profile (dotted line) is duplicated from Fig. 5. [See the electronic edition of the Journal for a color version of this figure.]

nicutt 2001). The surface density of $\mathrm{H}$ II regions declines abruptly near a galactocentric radius of $5 !^{\prime} 1\left(R_{\mathrm{H}}, 6.6 \mathrm{kpc}\right)$, suggesting that massive star formation is now confined to M83's inner disk, despite the prominence of a rather extended $\mathrm{H}$ I disk (Rogstad et al. 1974; Huchtmeier \& Bohnenstengel 1981; see also Fig. 1). Our GALEX observations modify this view. While the distribution of UV-bright stellar complexes is highly concentrated in the area occupied by $\mathrm{H}$ II regions (tracing $\mathrm{O}$ stars), many UV sources (tracing $\mathrm{O}$ and $\mathrm{B}$ stars) are easily recognizable far beyond the "edge" of this distribution. Furthermore, diffuse UV emission is also detected beyond the optically bright star-forming disk.

\subsection{Outer-Disk, UV-bright Stellar Complexes}

Our GALEX observations of M83 revealed more than 100 UV-bright star-forming regions and stellar clusters beyond $R_{\mathrm{H} \text { Ir }}$ Figure 1 shows that the outer-disk star-forming regions appear preferentially located on local maxima or filaments in the structure of the warped $\mathrm{H}$ I disk. The distribution of GALEX sources extends to the limit of the field surveyed at the VLA by Tilanus \& Allen (1993). We suspect that additional M83 star-forming regions may be located in the remainder of the $\mathrm{H}$ I envelope (Huchtmeier \& Bohnenstengel 1981), but this is challenging to confirm without corroborating $\mathrm{H}$ I morphology.

Upon inspection of POSS2 images, we found that the majority of the outer-disk GALEX detections could be discerned in IIIaJ+GG 385 (blue) plates. However, the apparent contrast of the sources is lower in the visible than at UV wavelengths. An undetermined fraction of the outer-disk star-forming complexes were discovered by Wamsteker et al. (1983) in their analysis of deep red (127-04+RG 630) Schmidt plates. To our 
knowledge, this is the only mention of the outer-disk sources in this galaxy prior to the GALEX data.

Figure 2 presents the FUV luminosity function for M83 starforming complexes in various disk locations. The outer-disk complexes (dashed line) appear systematically fainter than their innerdisk counterparts (dotted line). The median FUV luminosity of the inner-disk population is a factor of 3 greater than the corresponding figure beyond $R_{\mathrm{H} \text { II }}$ (only correcting for foreground extinction). Internal extinction is likely more severe in the inner disk. Thus, the intrinsic luminosity difference may be even higher than that shown in Figure 2, although we caution that blending and photometric incompleteness (more significant at $R<R_{\mathrm{H}}$ ) would have an opposite effect. The shape of the apparent FUV LF differs between the inner- and outer-disk populations. Our M83 FUV luminosity function of outer-disk stellar complexes is steeper than for the inner-disk population. A similar trend was noted in NGC 628 for the $\mathrm{H} \alpha \mathrm{LF}$ of $\mathrm{H}$ II regions (Lelievre \& Roy 2000). Because our GALEX measurements represent the aggregate flux of more than one independent cluster (or simply an extended distribution of massive stars), it is premature to interpret the precise slope of these FUV LFs in terms of the CMF. Blending tends to flatten observed LFs, but the magnitude of this effect may vary with environment. High-resolution follow-up observations would resolve ambiguities related to the FUV LF and median luminosity estimates. Nevertheless, the maximum UV luminosity exhibited by the population of outer-disk sources implies an upper limit of about $10^{5} M_{\odot}$ for the most recently formed complexes (see also Fig. 3).

In Figure 3 we compare the observed (NUV, FUV - NUV) color-magnitude diagram (CMD) for UV-bright complexes with population synthesis models of Bruzual \& Charlot (2003). M83's outer-disk sources have FUV - NUV colors generally consistent with ages from a few megayears up to $400 \mathrm{Myr}$ and are generally less massive than sources with $R<R_{\mathrm{H} \text { II }}$.

Only a subset of the outer-disk UV-bright sources have detected counterparts in $\mathrm{H} \alpha$ imagery. This effect could be partially due to a combination of limited sensitivity and the lower expected emission measure of $\mathrm{H}$ II nebulae in a low-density environment, but it supports the view that our GALEX observations trace a diverse population comprised of both young and moderate-age complexes. An important advantage of UV observations is the ability to directly trace stellar, rather than nebular, emission.

Figure 4 presents the distribution of total gas surface density at the locations of all inner (outer) disk sources using a dotted (dashed) line. The local surface densities at which outer-disk starforming complexes appear to be forming are typically subcritical with respect to the disk instability criterion (Martin \& Kennicutt
2001). However, we caution that the outer-disk measurements of $\Sigma_{\text {gas }}$ are lower limits in two important ways. Most obviously, the estimate of surface density at positions beyond $\sim R_{\mathrm{H} \text { II }}$ is based only on $\mathrm{H}$ I observations, neglecting the contribution from molecular gas traced by CO (unmapped outside of $14^{\prime} \times 14^{\prime}$ ). Also, the resolution of the $\mathrm{H}$ I aperture synthesis map is coarse in comparison to the scale of our detections (50" vs. $\left.5^{\prime \prime}\right)$. Higher resolution $21 \mathrm{~cm}$ observations could plausibly boost the apparent gas surface density in the local environment of the UV-bright complexes.

\subsection{Underlying Low Surface Brightness Disk}

The discrete outer-disk stellar complexes lie projected against a fainter, slowly varying field population. To place the UV-bright complexes in the context of M83's overall star formation history, we have plotted the median FUV, NUV, and $\mathrm{H} \alpha$ surface brightness (Fig. 5), plus FUV - NUV (Fig. 6), as a function of galactocentric radius. The underlying stellar population becomes substantially bluer (in FUV - NUV) with increasing radius. Dust is known to exist in the outer disks (and even in the extended $\mathrm{H}$ I disks) of spiral galaxies (Popescu \& Tuffs 2003) and thus is likely to be present in the outer disk of M83 too, particularly given the star formation traced there by GALEX. Accordingly, scattered light may contribute to the emission at each position (see also Popescu et al. 2005), tending to smooth the UV profile. Nevertheless, the FUV - NUV profile (Fig. 6) shows a sequence of breaks within the bright disk of M83. Outside of $R_{\mathrm{H} \text { I }}$ the FUV - NUV color becomes bluer almost monotonically, indicating that recent star formation dominates the UV emission. The median $\mathrm{H} \alpha$ surface brightness profile (Fig. 5.) is observed to drop off much more rapidly than either UV band. Given that the dynamical timescale beyond $R_{\mathrm{H} \text { II }}$ is $\gtrsim 60 \mathrm{Myr}$, the dropout of $\mathrm{H} \alpha$ emission compared to UV cannot likely be attributed to a (recent) coherent decrease in the star formation rate at large radii. FUV - NUV measurements for discrete sources are overplotted on Figure 6. Within $10^{\prime}$ galactocentric radius, the UV-bright star-forming regions generally appear bluer (younger) than or consistent with the FUV - NUV color of the underlying diffuse emission.

GALEX (Galaxy Evolution Explorer) is a NASA Small Explorer, launched in 2003 April. We gratefully acknowledge NASA's support for construction, operation, and science analysis for the GALEX mission, developed in cooperation with the Centre National d'Etudes Spatiales of France and the Korean Ministry of Science and Technology.

\section{REFERENCES}

Allen, R. J., Atherton, P. D., \& Tilanus, R. P. J. 1986, Nature, 319, 296

Bertin, E., \& Arnouts, S. 1996, A\&AS, 117, 393

Bianchi, L., et al. 2005a, in The Local Group as an Astrophysical Laboratory, ed. M. Livio (Baltimore: STScI), in press 2005b, ApJ, 619, L71

Boissier, S., Boselli, A., Prantzos, N., \& Gavazzi, G. 2005, ApJ, 619, L83

Bruzual, G., \& Charlot, G. 2003, MNRAS, 344, 1000

Crosthwaite, L. P., Turner, J. L., Buchholz, L., Ho, P. T. P., \& Martin, R. N. 2002, AJ, 123, 1892

Cuillandre, J.-C., Lequeux, J., Allen, R. J., Mellier, Y., \& Bertin, E. 2001, ApJ, 554, 190

de Blok, W. J. G., \& Walter, F. 2003, MNRAS, 341, L39

Ferguson, A. M. N., Gallagher, J. S., \& Wyse, R. F. G. 1998a, AJ, 116, 673

Ferguson, A. M. N., Wyse, R. F. G., Gallagher, J. S., \& Hunter, D. A. 1998b, ApJ, 506, L19

Harris, J., Calzetti, D., Gallagher, J. S., III, Conselice, C. J., \& Smith, D. A. 2001, AJ, 122, 3046
Huchtmeier, W. K., \& Bohnenstengel, H.-D. 1981, A\&A, 100, 72

Kennicutt, R. C., Jr. 1989, ApJ, 344, 685

Lelievre, M., \& Roy, J.-R. 2000, AJ, 120, 1306

Martin, C. L., \& Kennicutt, R. C., Jr. 2001, ApJ, 555, 301

Popescu, C. C., \& Tuffs, R. J. 2003, A\&A, 410, L21

Popescu, C., et al. 2005, ApJ, 619, L75

Rogstad, D. H., Lockart, I. A., \& Wright, M. C. H. 1974, ApJ, 193, 309

Sellwood, J. A., \& Balbus, S. A. 1999, ApJ, 511, 660

Smith, D. A., Allen, R. J., Bohlin, R. C., Nicholson, N., \& Stecher, T. P. 2000, ApJ, 538, 608

Thim, F., Tammann, G. A., Saha, A., Dolphin, A., Sandage, A., Tolstoy, E., \& Labhardt, L. 2003, ApJ, 590, 256

Tilanus, R. P. J., \& Allen, R. J. 1993, A\&A, 274, 707

Wamsteker, W., Lorre, J. J., \& Schuster, H. E. 1983, in IAU Symp. 100, Internal Kinematics and Dynamics of Galaxies, ed. A. Athanassoula (Dordrecht: Reidel), 65 